Military Technical College

Kobry El-Kobbah,

Cairo, Egypt.

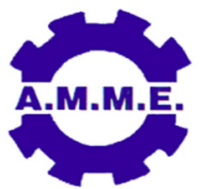

$16^{\text {th }}$ International Conference on Applied Mechanics and Mechanical Engineering.

\title{
ANALYTICAL INVESTIGATIONS OF THE GRAVITY EFFECT ON THE DYNAMICS AND PERFORMANCE OF CENTRIFUGAL PENDULUM VIBRATION ABSORBER
}

\author{
E. Abouobaia*, R. Bhat ${ }^{* \star}$ and R. Sedaghati ${ }^{* \star *}$
}

\begin{abstract}
Centrifugal Pendulum Vibration Absorber (CPVA) has been effectively used in rotating and reciprocating machines to control torsional vibrations passively. This research presents results from analytical investigation of rotor systems integrated with CPVA. The system under investigation is modeled as a rotating disk attached with CPVA in which the disk is subjected to an external simple harmonic torque. Mathematical model of a rotor fitted with a CPVA has been formulated and the equations of motion are derived using Lagrange principle. The effect of gravity due to CPVA operating in a vertical plane with a horizontal shaft has been considered in the formulation. The results are also compared with those based on CPVA operating in a horizontal plane in which the effect of gravity has been neglected. Moreover, softening nonlinear behavior of the pendulum and its effect on the natural frequency of the system has been investigated.
\end{abstract}

\section{KEY WORDS}

Torsional vibration absorber, CPVA, gravity effect, softening behavior.

\footnotetext{
* Graduate student, Dept. of Mech. \& Ind. Eng., Concordia University, Montreal, Canada.

** Professor, Dept. of Mech. \& Ind. Eng., Concordia University, Montreal, Canada.

*** Professor, Dept. of Mech. \& Ind. Eng., Concordia University, Montreal, Canada.
} 


\section{NOMENCLATURE}

$\begin{array}{ll}\theta \ldots . . . & \text { Rotor angular displacement } \\ \phi \ldots . . & \text { Pendulum angular displacement } \\ \psi \ldots . . & \text { Shaft torsional displacement } \\ \Omega \ldots . . & \text { Rotor rotational speed } \\ \omega_{r n . .} & \text { Shaft system natural frequency } \\ \omega_{a n . .} & \text { Absorber natural frequency } \\ \omega \ldots . . & \text { Excitation frequency } \\ \text { J.... } & \text { Rotor mass moment of inertia } \\ \text { T..... } & \text { Kinetic energy } \\ \text { V.... } & \text { Potential energy }\end{array}$

$\begin{array}{ll}\mathrm{R} . . . . & \text { Rotor radius } \\ \mathrm{C}_{\mathrm{r} . . .} & \text { Shaft viscous damping } \\ \mathrm{C}_{\ldots} . . . & \text { Absorber viscous damping } \\ T_{e \ldots .} & \text { Excitation torque } \\ T_{0 . . .} & \text { Torque amplitude } \\ \mathrm{m} . . . & \text { Pendulum mass } \\ g_{\ldots . . .} & \text { Gravitational acceleration } \\ V_{m} \ldots . & \text { Pendulum mass velocity } \\ l_{\ldots . . .} & \text { Pendulum length } \\ k \ldots . . & \text { Shaft torsional stiffness }\end{array}$

\section{INTRODUCTION}

Rotating machinery systems experience torsional vibrations to some degree during operation. Analysis of torsional vibration response of rotating machinery is an important consideration in defining the operational reliability of rotating parts. Excessive torsional vibrations may result in noise, excessive stresses or even fatigue failure. Some measures have been introduced to reduce torsional vibrations in rotating machinery, such as addition of flywheels and tuned vibration dampers [1-6]. However, these methods have some deficiencies. The flywheels increase the system inertia, and results in reduction in system responsiveness, while torsional dampers are effective at only a single frequency [7].

Centrifugal pendulum vibration absorber (CPVA) is one of the effective devices used for reducing torsional vibrations and are widely used in rotating and reciprocating machines to suppress torsional vibrations. These absorbers consist of a pendulum constrained to move in a plane normal to the rotational axis of the machine. CPVAs have been successfully employed to eliminate torsional vibrations in geared radial aircraft engine propeller systems [8], helicopter rotors [9-10], and also in some high performance automotive engines [11] in order to counteract the torsional vibrations in the rotating system. By proper selection of the optimum suspension point for the pendulum and its effective length, the absorber can be tuned to suppress torsional vibrations in a range of operating speeds.

Pendulum absorbers have been in use over half a century, however, they still continue to be used in new applications, which experience severe torsional vibrations. Pendulum absorbers have some tempting features for applications; since they can be tuned to a given order of rotation rather than to a set frequency and therefore they are effective over a continuous range of rotating speeds [12], also including the fact that they dissipate very little energy, in addition; they can often designed such that no mass or rotating inertia is added to the crank shaft as achieved by Nester et al. [13] in automotive applications by replacing existing counterweights by CPVAs, which then serve for both balancing and torsional vibration reduction. 
The operating envelop of CPVA depends on the amount of the employed mass of the absorber. Often in some applications, the mass must be minimized as in aerospace. Den Hartog [12] conducted a linear analysis of the dynamic response of the CPVA and briefly discussed the characteristics of its large amplitude nonlinear motion. Haddow and Shaw [14] established a rigid rotor fitted with several point mass absorbers that move along prescribed paths relative to the rotor. An external excitation torque is applied to the rotor that fluctuates at a given order of rotation and the absorber paths are selected such that their motion counteracts the applied torque.

From the available literature it has been shown that until around 1980 all CPVA designs employed simple circular paths for the absorbers [7]. Absorbers with circular paths work fine at small amplitudes; however, their nonlinear behavior due to amplitude dependent frequency limits their effectiveness. Absorbers with circular paths exhibit softening nonlinear behavior in which their natural frequency decreases as the amplitude increases. To overcome this shortcoming in the circular path absorbers, a little mistuning must be intentionally used to ensure stable and reliable operation of the CPVA.

Subsequently, various kinds of pendulum have been employed. In 1992, Denman [15] introduced other paths that offer improved performance for the absorbers at large amplitudes. Cycloidal path absorbers exhibit hardening nonlinear behavior; that is, the absorber natural frequency increases as the amplitude increases. Accordingly, Cycloidal absorbers offer improved performance at large amplitudes than that of a circular path. Another special absorber path is the epicycloidal path, since it maintains a constant frequency for the absorber over all amplitudes, thus keeping the absorber as linear as possible over a large operating range; this type is typically used in automotive engines [16]. In order to overcome limitation of passive vibration absorbers, Chen et al. [17] developed an active dynamic absorber to retain the advantages of the conventional CPVA. The active pendulum absorber is simply a CPVA connected to a torque motor that acts as an actuator to drive the pendulum at adequate oscillatory amplitude. It has very wide range of operating frequencies. This active absorber can be tuned according to the system characteristics to meet desired requirements. Genetic Algorithm controller for the active pendulum absorber was presented by Liang and Tung [18]. The proposed algorithm estimates an appropriate value for the torque of the active pendulum that is capable of suppressing the torsional vibration of the carrier.

Ryan and Shaw [19] studied the transient response characteristics of a single absorber fitted to a rotor that is subjected to engine order torsional excitation, two types of absorber were considered; Circular path and Tautochronic absorber. An approximate analytical method was developed for predicting the transient overshoot of CPVA when the rotor is subjected to near resonant torsional excitation. Although much work has been done in this field, past research has focused mainly on analysis of absorbers operating in horizontal plane, which does not experience the gravity effect. In the present study, the rotor system integrated with the pendulum absorber has been investigated in both vertical and horizontal planes in order to assess the effect of gravity. 
The system under investigation is modeled as a rotating disk attached with CPVA in which the disk is subjected to an external harmonic torque. Mathematical modeling of a CPVA has been formulated using Lagrange principle, resulting in two coupled nonlinear differential equations of motion for both rotor and the pendulum. The results obtained from numerical analysis for both rotor and absorber responses are illustrated for each case and compared against one another. In addition, softening nonlinear behavior of the pendulum and its effect on the natural frequency of the system has been investigated.

\section{EQUATIONS OF MOTION}

Lagrangian formulation is used to model the rotor integrated with CPVA in which two coupled nonlinear equations are derived for the rotor and the pendulum. The kinetic energy for the rotor and CPVA system shown in Fig. 1 is given by:

$$
T=\frac{1}{2} m v_{m}^{2}+\frac{1}{2} J \dot{\theta}^{2}
$$

where $v_{m}$ is the velocity vector of the pendulum and $J$ is the mass moment of inertia of the rotor. The potential energy is given by:

$$
V=m g[R \sin \theta+l \sin (\theta+\phi)]
$$

The total velocity of the pendulum with respect to the point $O$ is obtained as:

$$
v_{m}^{2}=2 l(R \cos \phi+l) \dot{\theta} \dot{\phi}+\left(R^{2}+l^{2}+2 R l \cos \phi\right) \dot{\theta}^{2}+l^{2} \dot{\phi}^{2}
$$

Substituting Eq. (3) into Eq. (1) yields:

$$
T=\frac{1}{2} m\left[2 l(R \cos \phi+l) \dot{\theta} \dot{\phi}+\left(R^{2}+l^{2}+2 R l \cos \phi\right) \dot{\theta}^{2}+l^{2} \dot{\phi}^{2}\right]+\frac{1}{2} J \dot{\theta}^{2}
$$

The total rotation $(\theta)$ of the rotor system consists of two parts, a steady rotation $(\Omega t)$ due to rotation at a constant angular speed $(\Omega)$, and a small torsional oscillation $\psi(t)$ about this rotation and is given by:

$$
\theta=\Omega t+\psi(t)
$$

Substituting the value of $(\theta)$ from Eq. (5) into Eq. (4), the kinetic energy becomes:

$$
\begin{aligned}
T=\frac{1}{2} m[2 l(R & \left.\cos \phi+l)(\Omega+\dot{\psi}) \dot{\phi}+\left(R^{2}+l^{2}+2 R l \cos \phi\right)(\Omega+\dot{\psi})^{2}+l^{2} \dot{\phi}^{2}\right] \\
+ & \frac{1}{2} J(\Omega+\dot{\psi})^{2}
\end{aligned}
$$

Substituting Eq. (5) into Eq. (2) and adding the shaft strain energy, the potential energy becomes: 


$$
V=m g[R \sin (\Omega t+\psi)+l \sin (\Omega t+\psi+\phi)]+\frac{1}{2} k \psi^{2}
$$

where $k$ is the shaft stiffness. The Lagrangian [ $L=T-V$ ] in terms of the two generalized coordinates $\psi$ and $\phi$ is given by:

$$
\begin{gathered}
L=\frac{1}{2} J(\Omega+\dot{\psi})^{2}+\frac{1}{2} m\left[2 l(R \cos \phi+l)(\Omega+\dot{\psi}) \dot{\phi}+\left(R^{2}+l^{2}+2 R l \cos \phi\right)(\Omega+\dot{\psi})^{2}+l^{2} \dot{\phi}^{2}\right] \\
-m g[R \sin (\Omega t+\psi)+l \sin (\Omega t+\psi+\phi)]-\frac{1}{2} k \psi^{2}
\end{gathered}
$$

The equations of motion are given by:

$$
\begin{gathered}
\frac{d}{d t}\left(\frac{\partial L}{\partial \dot{\psi}}\right)-\frac{\partial L}{\partial \psi}=T_{e}-c_{r} \dot{\psi} \\
\frac{d}{d t}\left(\frac{\partial L}{\partial \dot{\phi}}\right)-\frac{\partial L}{\partial \phi}=-c_{a} \dot{\phi}
\end{gathered}
$$

where $T_{e}$ is the excitation torque, $C_{r}$ and $C_{a}$ are viscous damping coefficients for shaft system and absorber, respectively.

Substituting Eq. (8) into (9) and (10) results in the final equations of motion as:

$$
\begin{gathered}
\left(J+m l^{2}+m R^{2}+2 m R l \cos \phi\right) \ddot{\psi}+\left(m l^{2}+m R l \cos \phi\right) \ddot{\phi}-(2 m R l \sin \phi)(\dot{\psi}+\Omega) \dot{\phi} \\
-(m R l \sin \phi) \dot{\phi}^{2}+m g[R \cos (\psi+\Omega t)+l \cos (\psi+\Omega t+\phi)]+k \psi=T_{e}-C_{r} \dot{\psi} \\
\left(m l^{2}+m R l \cos \phi\right) \ddot{\psi}+m l^{2} \ddot{\phi}+m R l \sin \phi(\dot{\psi}+\Omega)^{2}+m g l \cos (\psi+\Omega t+\phi)=-C_{a} \dot{\phi}
\end{gathered}
$$

It should be noted that when the absorber operates in the Horizontal Plane, there is no gravity effect. Hence the potential energy in Eq. (7) will be due to the shaft strain energy alone as follows:

$$
V=\frac{1}{2} k \psi^{2}
$$

The Lagrangian [ $L=T-V$ ] for the two generalized coordinate $\psi$ and $\phi$ will then become:

$$
\begin{gathered}
L=\frac{1}{2} J(\Omega+\dot{\psi})^{2}+\frac{1}{2} m\left[2 l(R \cos \phi+l)(\Omega+\dot{\psi}) \dot{\phi}+\left(R^{2}+l^{2}+2 R l \cos \phi\right)(\Omega+\dot{\psi})^{2}+\right. \\
\left.l^{2} \dot{\phi}^{2}\right]-\frac{1}{2} k \psi^{2}
\end{gathered}
$$

Substituting of Eq. (14) into (9) and (10) results in two equations of motion same as Eq. (11) and (12) without the terms that contain (g) [19]. 
Assuming small oscillations of the pendulum we have $\sin \phi \approx \phi, \cos \phi \approx 1$ and neglecting the quadratic nonlinearities in Eqs. (11) and (12) then solving for the natural frequencies of the system yields [20]:

- Shaft system natural frequency $\left(\omega_{r n}\right)$ :

$$
\begin{gathered}
J \ddot{\psi}+k \psi=0 \\
\omega_{r n}=\sqrt{\frac{k}{J}}
\end{gathered}
$$

- Absorber natural frequency $\left(\omega_{a n}\right)[20]$ :

$$
\begin{gathered}
m l^{2} \ddot{\phi}+m R l \Omega^{2} \phi=0 \\
\omega_{a n}=\Omega \sqrt{\frac{R}{l}}
\end{gathered}
$$

\section{RESULTS AND DISCUSSION}

This section presents results obtained from the numerical simulation of the rotor system with CPVA operated in both vertical and horizontal plane and results are compared.

\section{Solution of the Nonlinear Equations for the 2 DOF Systems:}

\section{Absorber operating in vertical plane}

Equations (11) \& (12) corresponding to the absorber operating in the Vertical Plane with $T_{e}=0$ (free vibration) are solved numerically. An initial angular velocity for the shaft system is assumed as $\dot{\psi}=15 \mathrm{rad} / \mathrm{sec}$. Table 1 provides the numerical values assigned to the other parameters. The FFT of the rotor response shown in Fig. 2, clearly illustrates the two nonlinear damped natural frequencies of the system, $\omega_{1}=$ $19 \mathrm{~Hz} \& \omega_{2}=113 \mathrm{~Hz}$, as the absorber operates in the Vertical Plane.

\section{Absorber operating in horizontal plane}

Equations (11) \& (12) without (g) terms corresponding to the absorber operating in the Horizontal Plane with $T_{e}=0$ (free vibration) are also solved numerically. The FFT of rotor response shown in Fig. 3 illustrates the two nonlinear damped natural frequencies, $\omega_{1}=20 \mathrm{~Hz} \& \omega_{2}=112 \mathrm{~Hz}$, as the absorber operates in the Horizontal Plane. By comparing the results obtained for the rotor system natural frequencies in both vertical and horizontal planes, one will find that the effect of gravity has a small contribution on both system natural frequencies. 


\section{Investigating Rotor Torsional Response}

Solving the two nonlinear differential equations (11) \& (12) numerically using Matlab Simulink the shaft system torsional response when absorber is operating in the vertical plane are obtained and are shown in Fig. 4.

By solving equations (11) \& (12) without (g) terms, the system torsional response can be obtained as shown in Fig. 5 when absorber is operating in the horizontal plane. The pendulum angular displacement responses for both vertical and horizontal planes are also shown in Fig. 6 and Fig. 7.

In the case of the shaft system operating without the absorber, the equation of motion will be as follows:

$$
J \ddot{\psi}+c_{r} \dot{\psi}+k \psi=T_{e}
$$

In Fig. 8 the shaft system torsional response when operating without absorber is illustrated and can be obtained by solving equation (19) numerically. In all the three aforementioned cases, the excitation torque was assumed to be:

$$
T_{e}=T_{o} \sin \omega t
$$

It is important to investigate the rotor system behavior when operating at the critical speed at which the rotor speed coincides with the system natural frequency; at this condition the system has the maximum vibration amplitude. Hence, numerical simulation of the rotor system should be modeled such that

$$
\Omega=\sqrt{\frac{k}{J}}
$$

Also the shaft system is set to be excited with an excitation frequency equal to the system natural frequency:

$$
\omega=\sqrt{\frac{k}{J}}
$$

Applying the resonance tuning condition in order to achieve the best effective inertia of the pendulum, the pendulum design parameters must meet the following requirement [20]:

$$
\frac{\omega^{2}}{\Omega^{2}}=\frac{R}{l}
$$

From equations (21), (22) \& (23), the absorber should be designed such that:

$$
\mathrm{R}=l
$$


Fig. 4, Fig. 5 and Fig. 8 show the shaft torsional response when the rotor system operates in vertical plane, horizontal plane and operated without absorber, respectively. The shaft steady state response amplitude shown in Fig. 4 is $\left(5 \times 10^{-5}\right.$ rad) as the absorber operates in vertical plane, and is around $60 \%$ larger than that shown in Fig. 5 which is $\left(2 \times 10^{-5} \mathrm{rad}\right)$ for the absorber operating in horizontal plane.

The difference in the shaft torsional responses in the two cases is due to the gravity effect when the absorber operates in vertical plane; hence the pendulum weight contributes to the potential energy term, and results in an additional torsional load on the rotor which appears clearly in the shaft response in Fig. 4. Consequently, gravity effect is an important consideration in defining the operational reliability in some important applications such as in Aerospace applications. In contrast, pendulum angular displacement amplitude when operating in both vertical and horizontal planes illustrated in Fig. 6 \& Fig. 7, show that there is no change in both amplitudes when the absorber is operating either in vertical or horizontal plane.

As shown in Fig. 8, the rotor steady state torsional response amplitude approaches $(0.1 \mathrm{rad})$ as the shaft system operates without absorber. When the absorber is attached to the rotor system, the amplitude is significantly reduced to $\left(2 \times 10^{-5} \mathrm{rad}\right)$ when operating in horizontal plane and $\left(5 \times 10^{-5} \mathrm{rad}\right)$ when operating in vertical plane. This clearly shows that the absorber can effectively reduce large torsional vibrations of the shaft system especially when the absorber is tuned correctly to the resonance tuning condition.

\section{Investigating Rotor Torsional Amplitude at Different Operating Speed}

Rotor torsional response is investigated over the normal operating speed range (0$120 \mathrm{~Hz}$ ) in both vertical and horizontal plane. As shown in Fig. 9, at low operating speed range $(0-30 \mathrm{~Hz})$ the shaft system operating in vertical plane has torsional vibration amplitude much larger than that when operating in horizontal plane. However, as the rotor operating speed increases, the difference between both amplitudes decreases. In automotive applications, the obtained results serve as important guidelines in design stage since at low operating speed torsional vibrations transmitted from cylinders at every power stroke results in serious fatigue failure of the crank as well as significant side effect on other mechanisms if not effectively controlled. Consequently, it is important to take into account the gravity effect especially when the rotor system is operating at low speeds.

\section{Softening Behavior of the Nonlinear Pendulum}

It is clear from equations (18) \& (24) that the natural frequency of the pendulum should be equal to the rotor speed since the rotor radius is equal to the pendulum length in the present case. From the results it was shown that at rotor speed of $10 \mathrm{~Hz}$ the natural frequency was $10 \mathrm{~Hz}$, as seen in Fig. 10. However, the natural frequency at rotor speed $50 \mathrm{~Hz}$ was found to be $40 \mathrm{~Hz}$, as shown in Fig. 11 . 
Natural frequencies at different rotor speeds are plotted in Fig. 12. It is clear from the results obtained that the natural frequency varies with the rotor frequency due to the softening nonlinear behavior of the pendulum. As a result the natural frequency of the pendulum decreases when the rotor speed increases [7].

Hence, absorbers with circular paths exhibit softening nonlinear behavior when their frequency decreases as the amplitude increases. To overcome this shortcoming in the circular path absorbers, a little mistuning must be intentionally introduced to ensure stable and reliable operation of the CPVA.

\section{CONCLUSION}

In this research analytical investigation of rotor system fitted with centrifugal pendulum vibration absorber has been presented. Three cases have been investigated. In the first two cases the rotor system was attached with the pendulum absorber and operated in both vertical and horizontal planes. Then the rotor was operated individually without the absorber. Shaft system and absorber responses in each case were presented and compared against each other. From the numerical analysis results, for cases when the rotor attached with CPVA operated in either vertical or horizontal plane, it is seen that the CPVA can effectively reduce the rotor torsional vibrations. In addition, the effect of gravity in the potential energy has been investigated when the absorber operated in the vertical plane. The absorber softening nonlinear behavior has been investigated and results showed that the absorber frequency decreases as the amplitude of torsional vibration increases, which leads to reduction in absorber performance. To overcome this shortcoming in the circular path absorbers, a little mistuning must be intentionally used to keep the absorber as linear as possible over a large operating speed range.

\section{REFERENCES}

[1] Ker Wilson W., "Practical Solution of Torsional Vibration Problems", chapter III. Volume IV, Chapman and Hall Ltd, London, 3rd edition, (1968).

[2] Taylor F., "The Internal Combustion Engine in theory and Practice", Cambridge, Massachusetts, The MIT Press, (1985).

[3] Igusa T. and Xu K., "Vibration control using multiple tuned mass dampers", Journal of sound and vibration 175 (4), pp. 491-503, (1994).

[4] Joshi A. and Jangid S., "Optimum parameters of multiple tuned mass dampers for base-excited damped systems", Journal of sound and vibration, 202 (5), pp. 657-667, (1997). 
[5] Vonflotow H., Beard A. and Bailey D., "Adaptive tuned vibration absorbers: tuning laws, tracking agility, sizing, and physical implementations", Proceedings of the National Conference on Noise Control Engineering, vol. 1, pp. 437-454. (1994).

[6] Rahul R. and Soong T., "Parametric study and simplified design of tuned mass dampers", Engineering structures 20 (3), pp. 193-204, (1998).

[7] Alsuwaiyan S. and Shaw W., "Performance and Dynamic Stability of GeneralPath Centrifugal Pendulum Vibration Absorbers", Journal of Sound and Vibration, 252(5), pp. 791-815, (2002).

[8] Taylor S., "Eliminating crankshaft torsional vibration in radial aircraft engines", No. 360105. SAE Technical Paper, (1936).

[9] Hamouda M. and Pierce G., "Helicopter vibration suppression using simple pendulum absorbers on the rotor blade", Journal of American Helicopter Society, 29(3), pp.19-29, July (1984).

[10] Madden F., "Constant frequency bifilar vibration absorber", U.S. Patent 4,218,187, issued August 19, (1980).

[11] Borowski V., Denman H., Cronin D., Shaw W., Hanisko P., Brooks T., Milulec D., Crum W., and Anderson P., "Reducing vibration of reciprocating engines with crankshaft pendulum vibration absorbers", SAE Technical Paper Series 911876, (1991).

[12] Den Hartog J., "Mechanical Vibrations", Dover Publications, Inc. New York. (1985).

[13] Nester M., Haddow A., Shaw W., Brevick J. and Borowski V., "Vibration reduction in a variable displacement engine using pendulum absorbers", In Proceedings of the SAE Noise and Vibration Conference and Exhibition, no. 2003-01, p. 1484, (2003).

[14] Haddow G. and Shaw W., "Centrifugal pendulum vibration absorbers: an experimental and theoretical investigation", Nonlinear Dynamics 34.3-4, pp. 293-307, (2003).

[15] Denman H, "Tautochronic Bifilar Pendulum Torsion Absorbers for Reciprocating Engines", Journal of Sound and Vibration, 159(2), pp. 251-277, (1992). 
[16] Lee T. and Shaw S., "Torsional vibration reduction in internal combustion engines using centrifugal pendulums", Proceedings of ASME Design Engineering Technical Conference, Vol. 3-Part A, pp. 487-492, (1995).

[17] Chen-Yi D., Chyun-Chau F. and Pi-Cheng T., "Application of voice coil motors in active dynamic vibration absorbers", Magnetics, IEEE Transactions on 41, no. 3, pp. 1149-1154, (2005).

[18] Liang C. and Tung P., "Application of genetic algorithms to active vibration control of a centrifugal pendulum vibration absorber", Proceedings of the Institution of Mechanical Engineers, Part I: Journal of Systems and Control Engineering, vol. 224, pp. 329-338, (2010).

[19] Ryan J., and Shaw S., "Nonlinear transient dynamics of pendulum torsional vibration absorbers", Journal of Vibration and Acoustics 135.1, 011017, (2013).

[20] Thomson W., "Vibration theory and applications", Englewood Cliffs, NJ, Prentice-Hall, (1991).

\section{LIST OF FIGUERS}

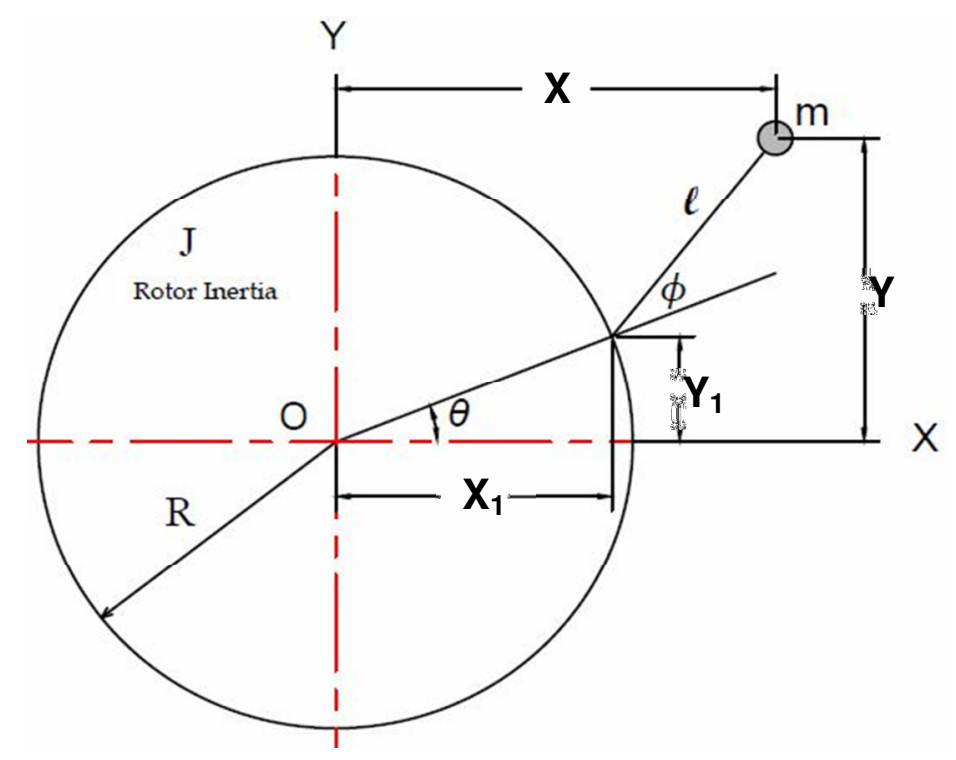

Fig. 1 Centrifugal Pendulum Absorber 


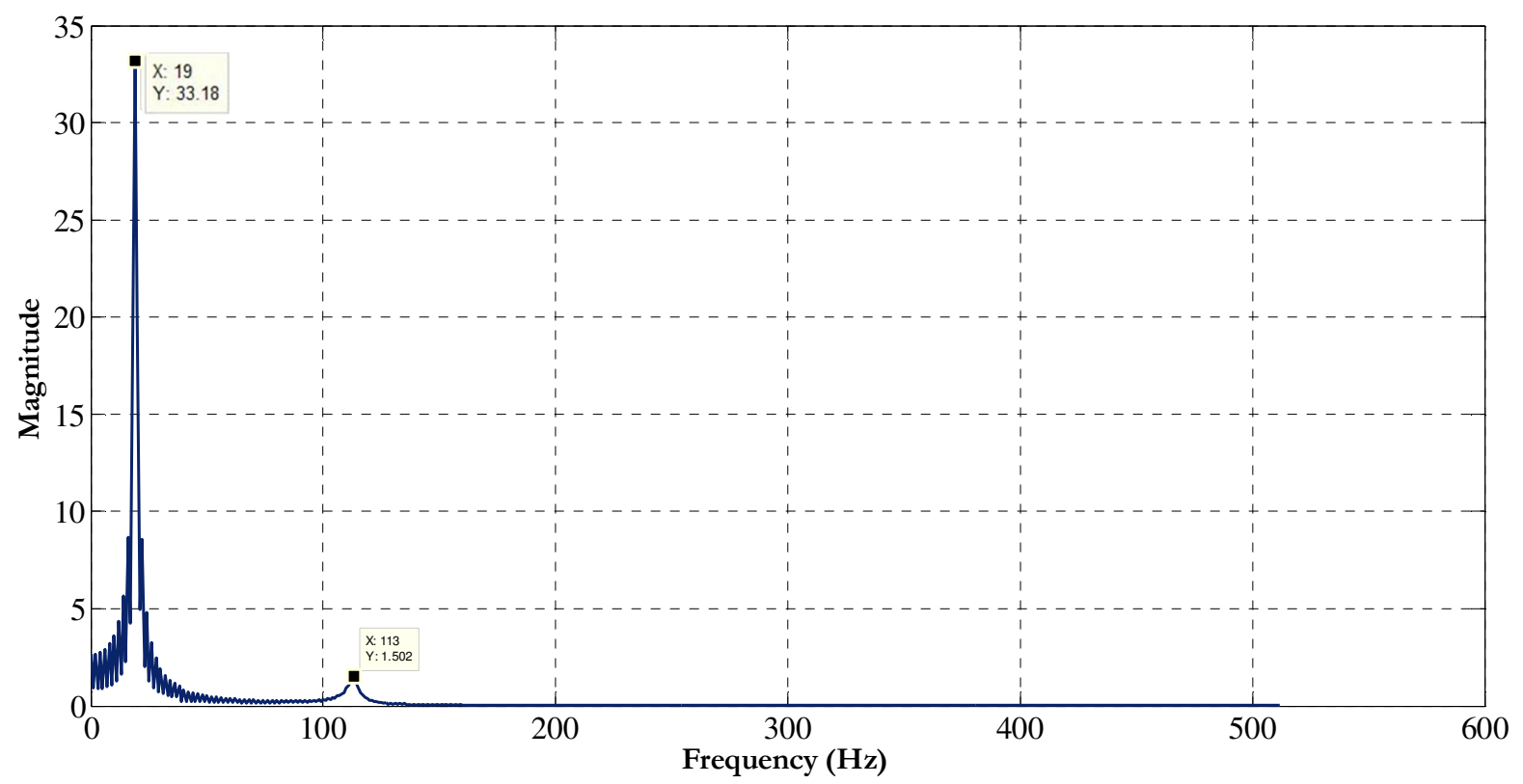

Fig. 2 Rotor FFT response when CPVA is operating in vertical plane

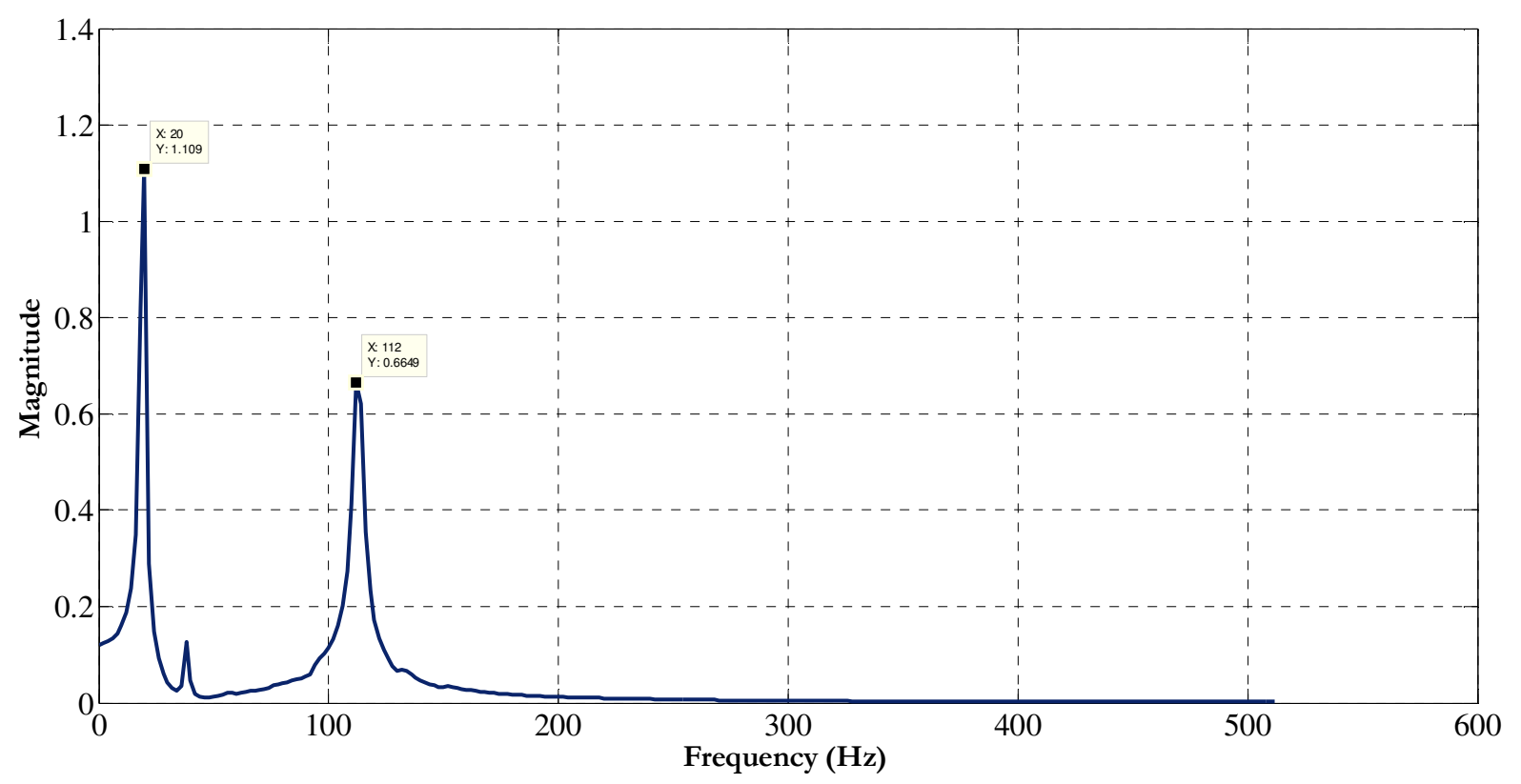

Fig. 3 Rotor FFT response when CPVA is operating in horizontal plane 


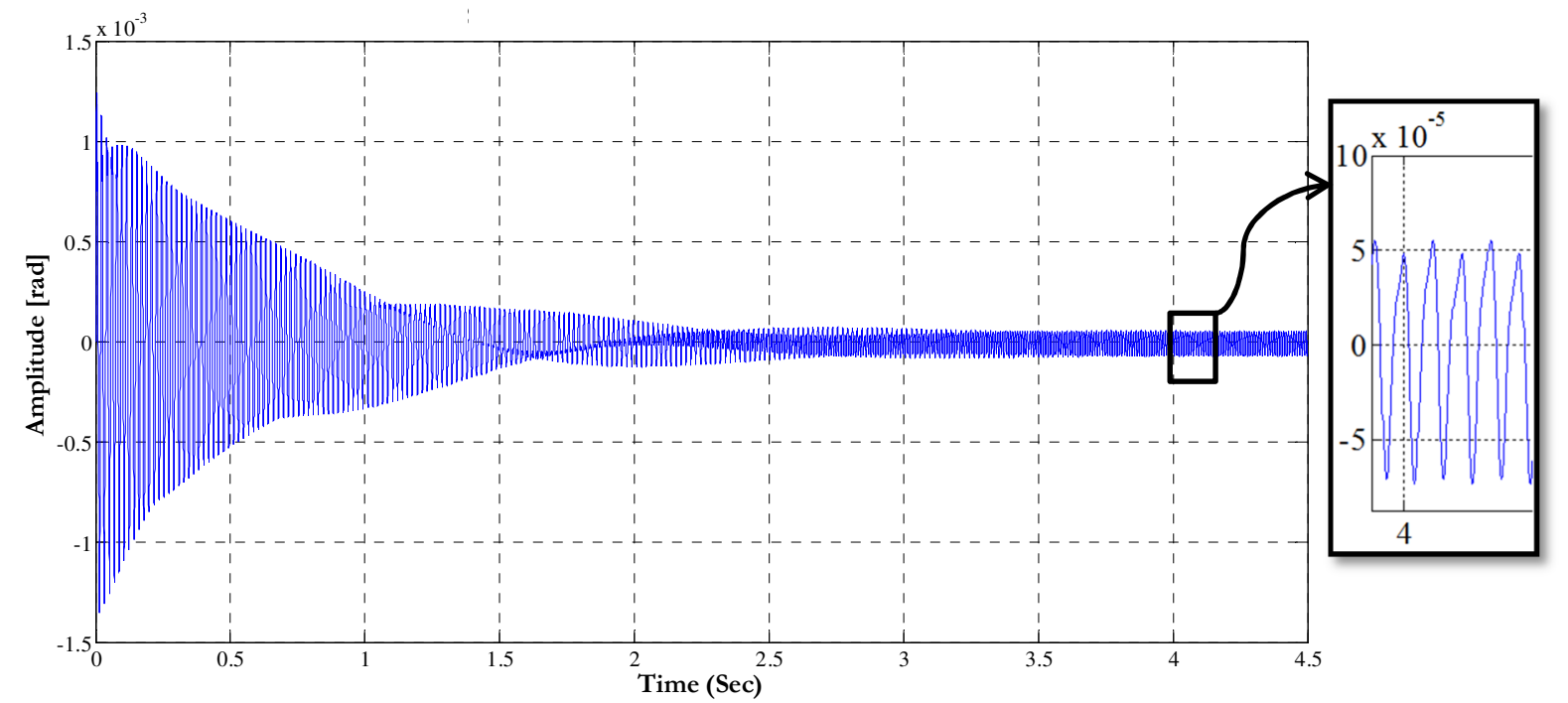

Fig. 4 Torsional response of the rotor when CPVA is operating in vertical plane

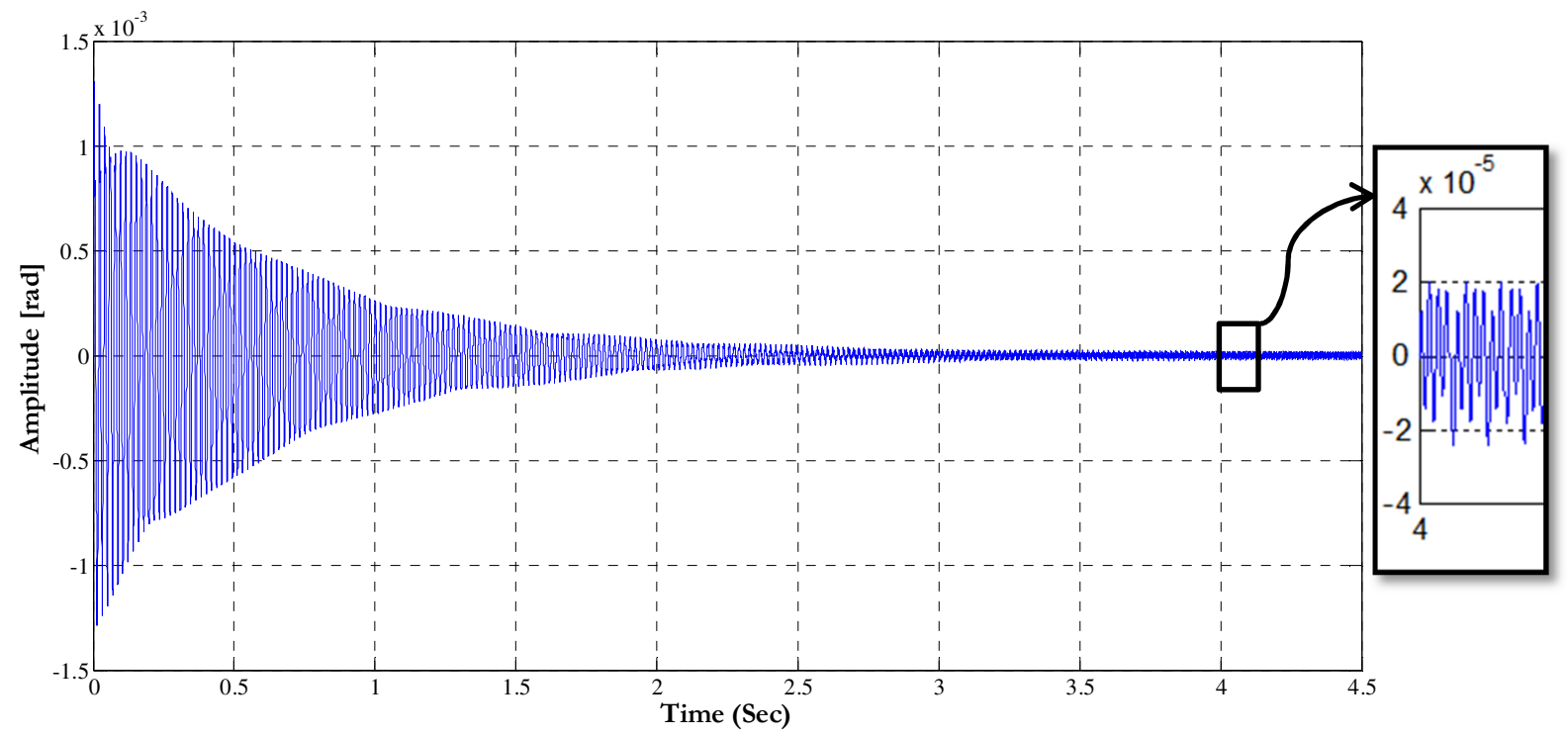

Fig. 5 Torsional response of the rotor when CPVA is operating in horizontal plane 


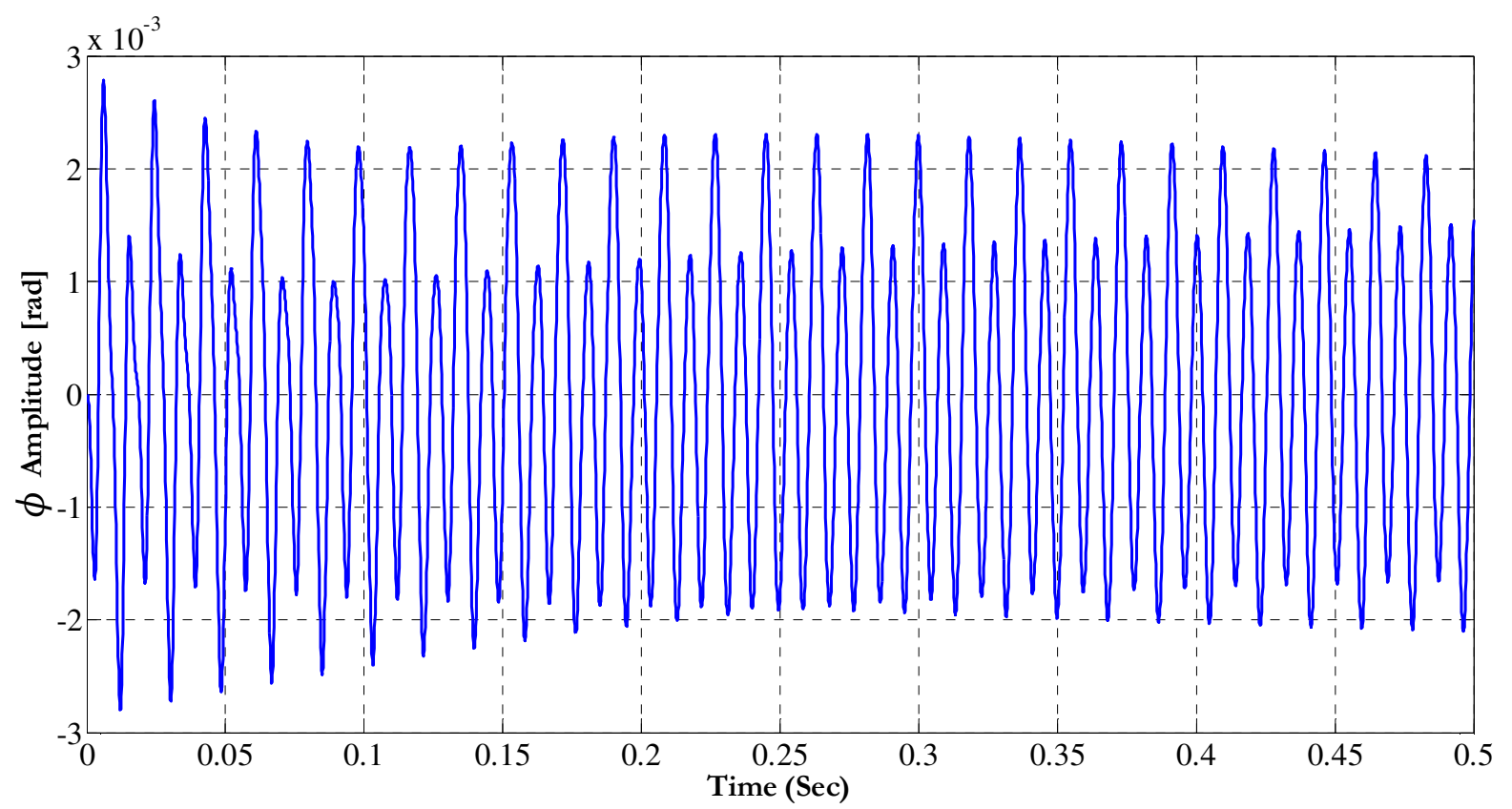

Fig. 6 Pendulum angular displacement amplitude operating in vertical plane

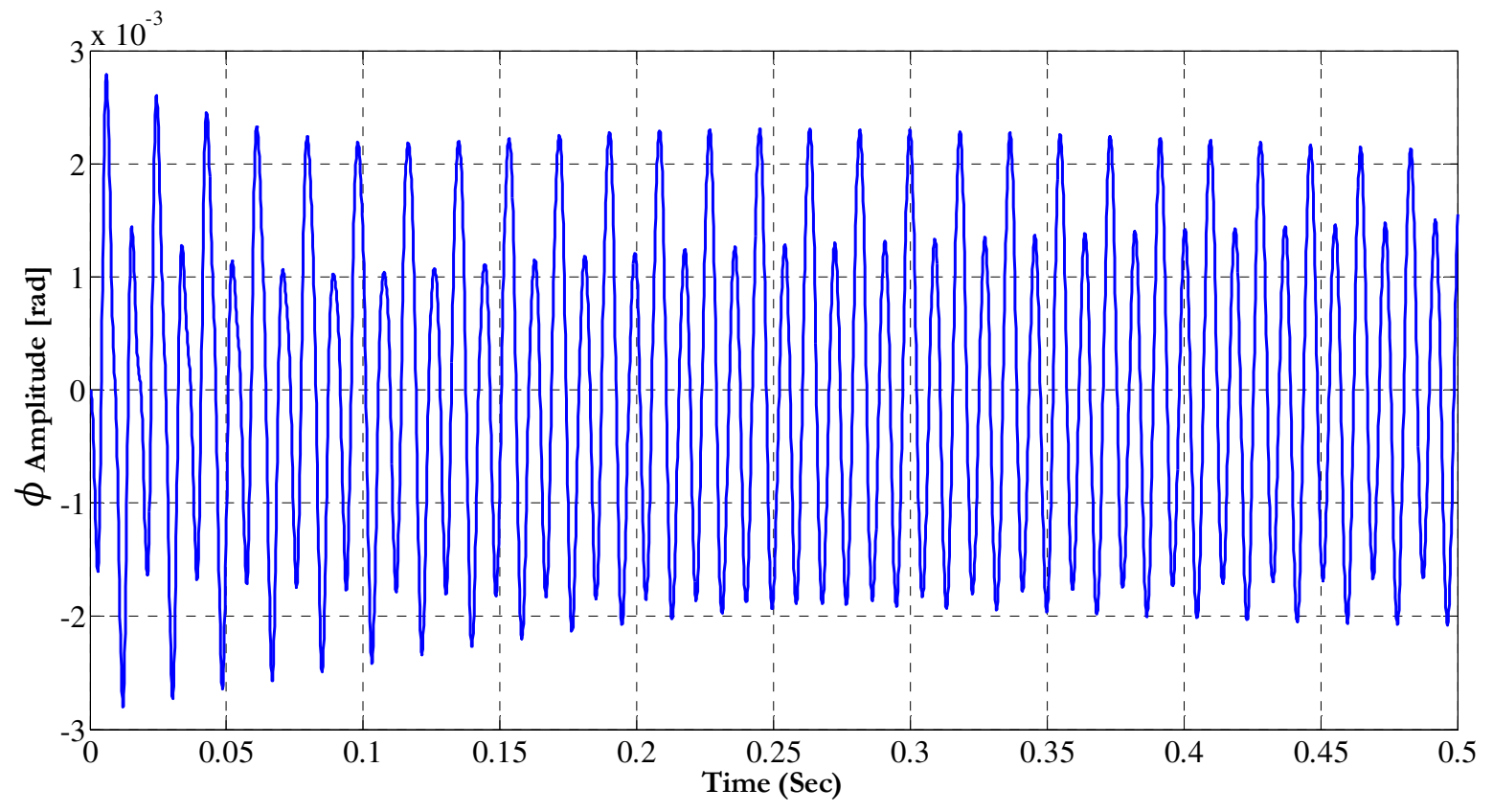

Fig. 7 Pendulum angular displacement amplitude operating in horizontal plane 

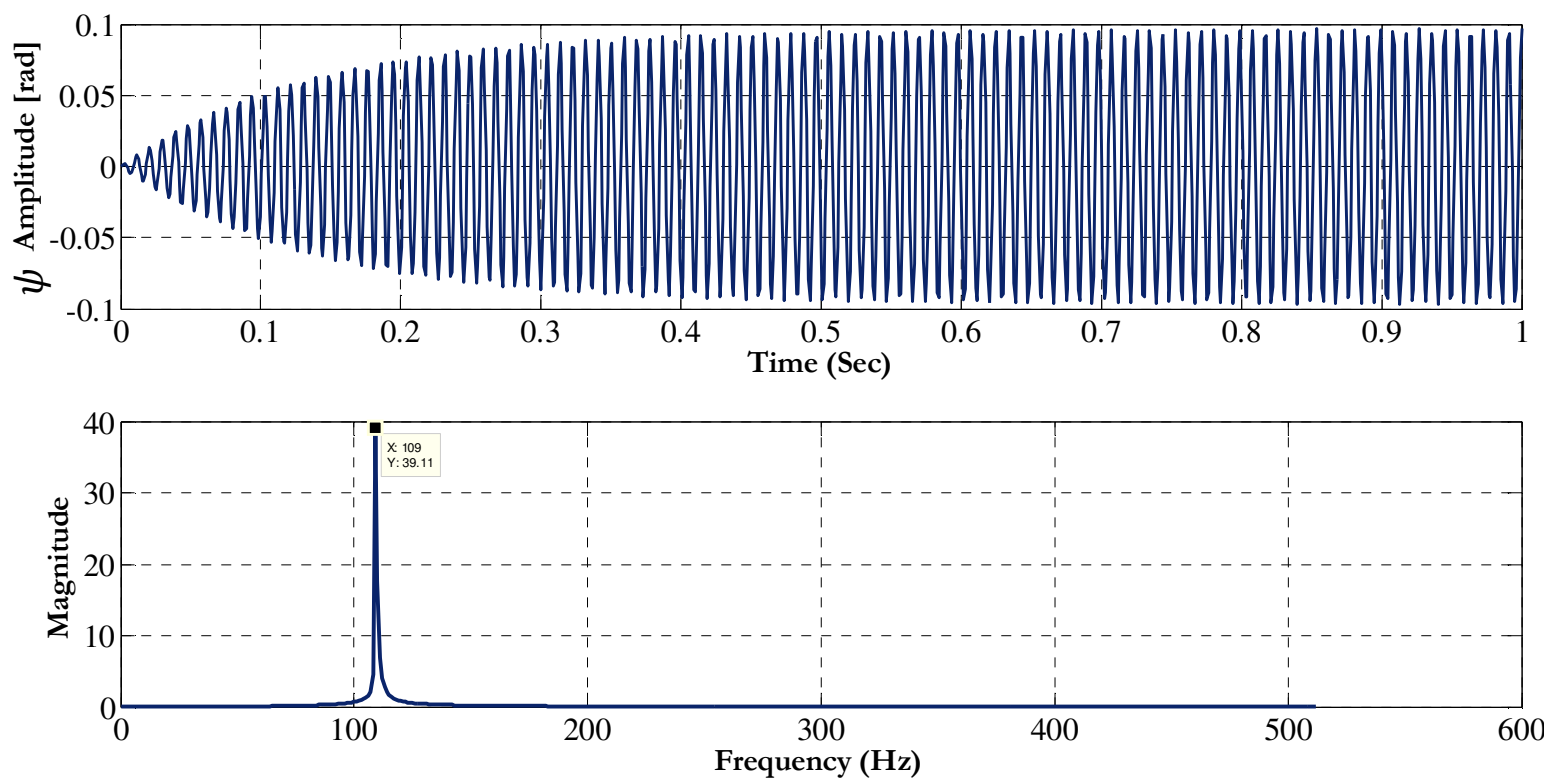

Fig. 8 Rotor torsional response operating without absorber

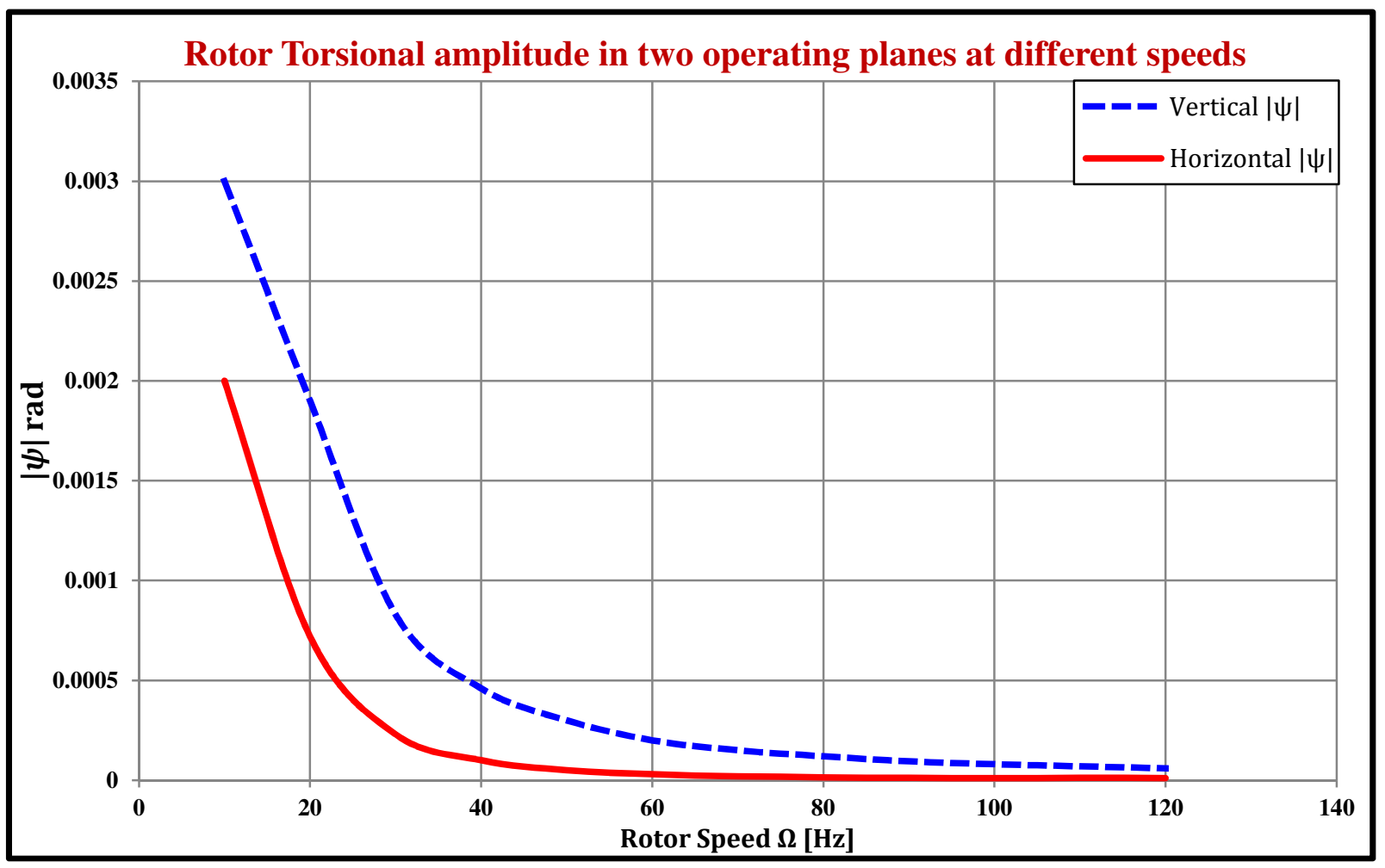

Fig. 9 Rotor torsional amplitude variation with rotor speed 


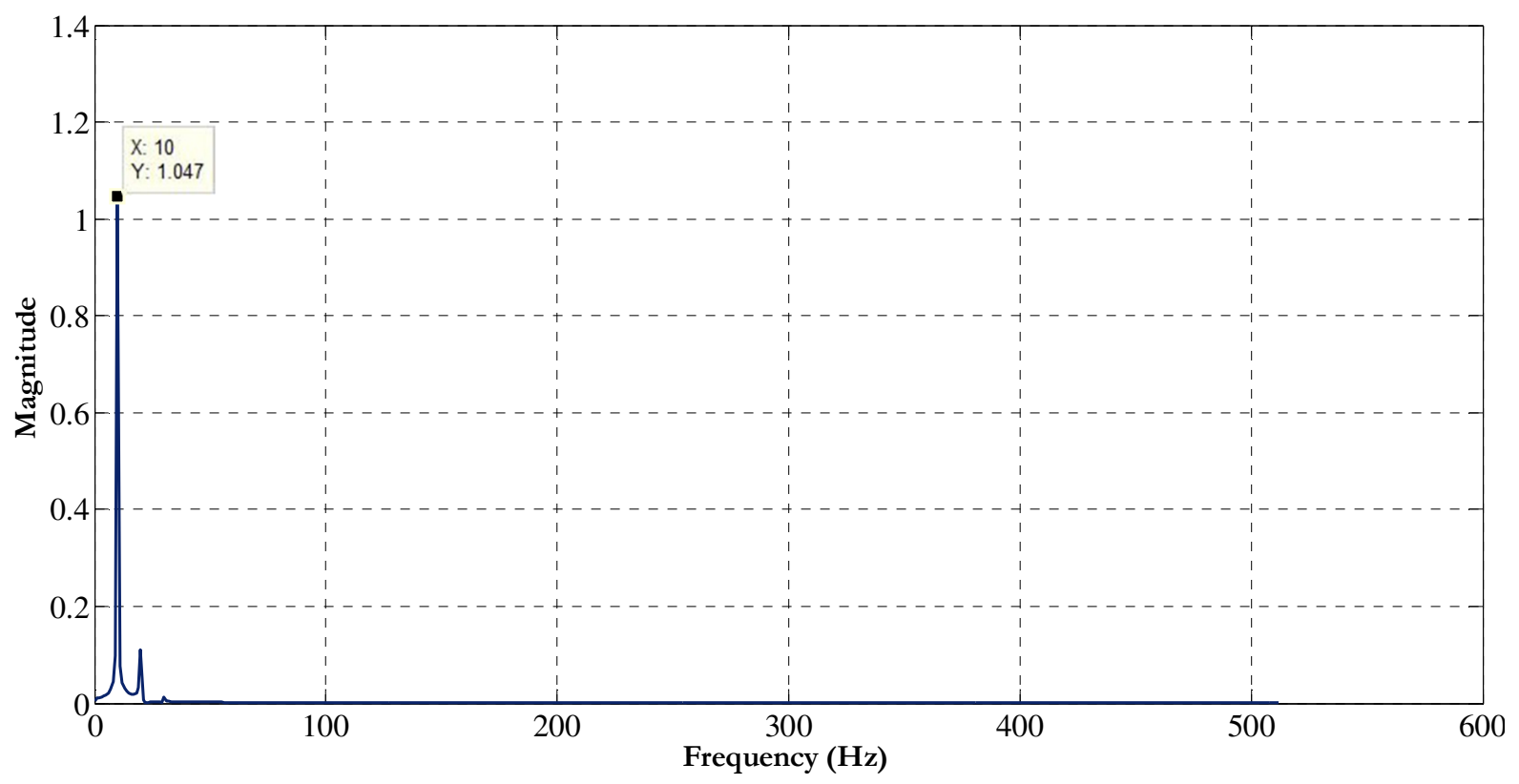

Fig. 10 Pendulum natural frequency at rotor speed $10 \mathrm{~Hz}$

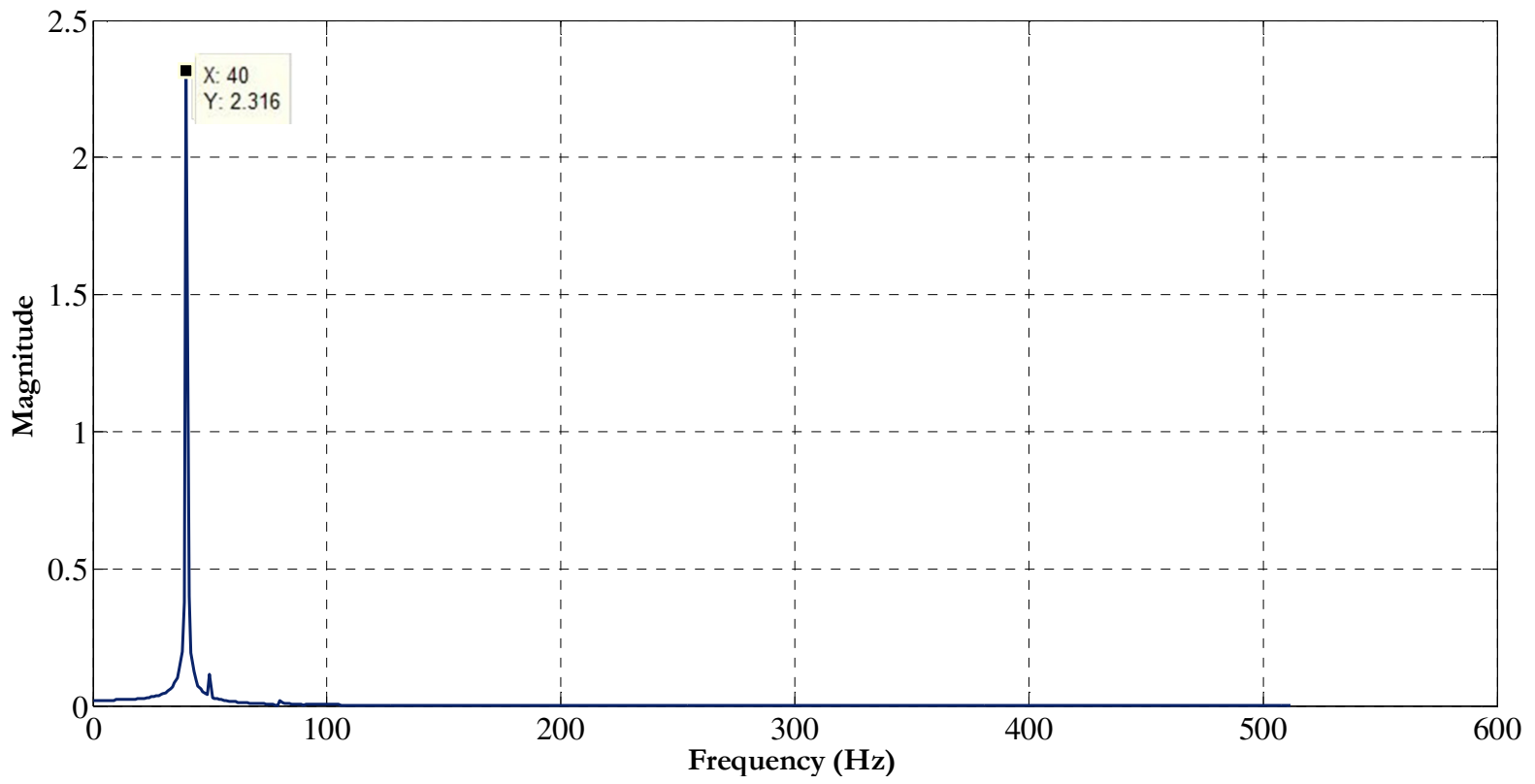

Fig. 11 Pendulum natural frequency at rotor speed $50 \mathrm{~Hz}$ 


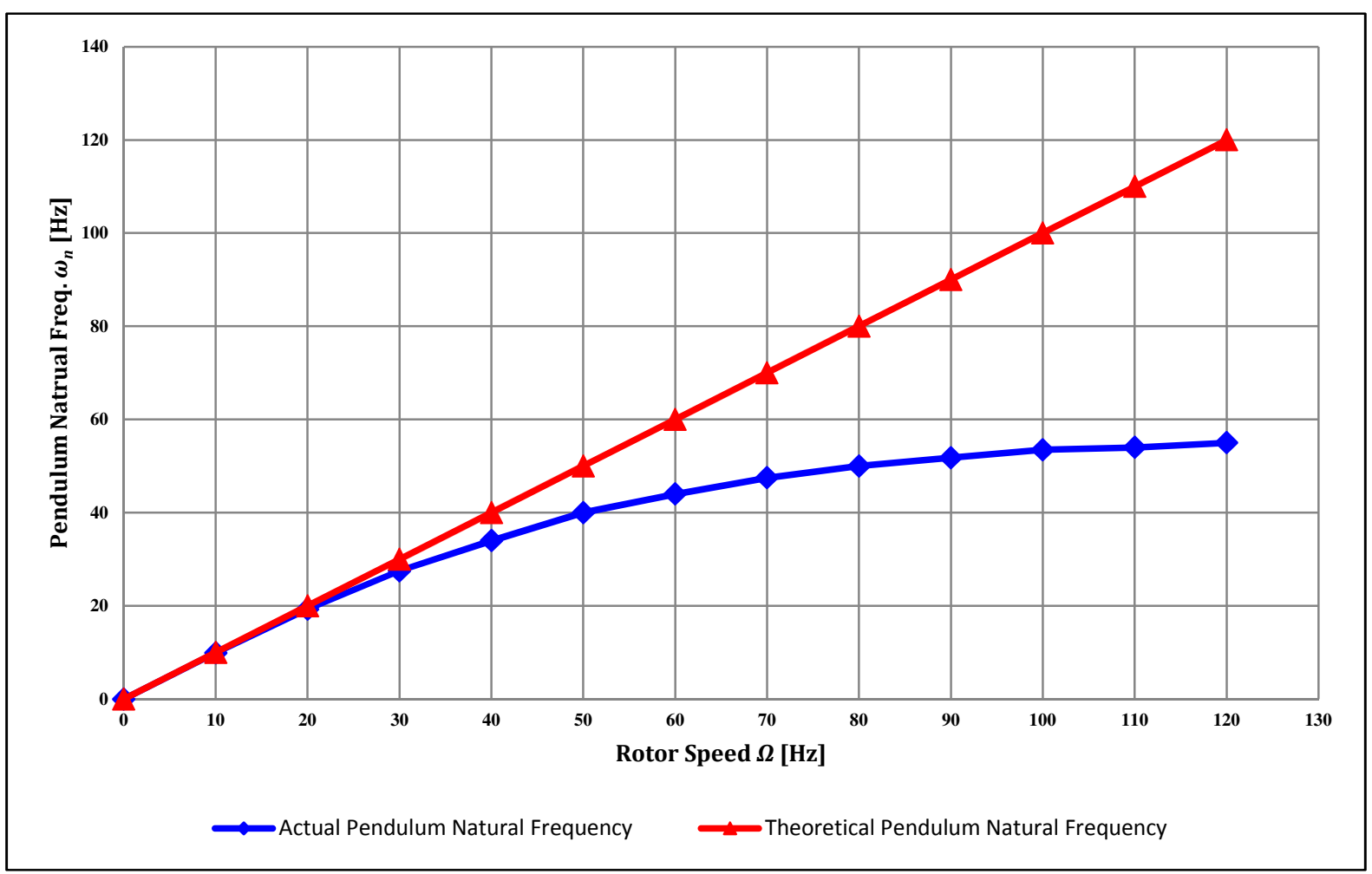

Fig. 12 Pendulum natural frequency variation with rotor speed

\section{LIST OF TABLES}

Table 1 Numerical values used in the analysis

\begin{tabular}{|l|c|c|c|}
\hline \multicolumn{1}{|c|}{ Name } & Parameter & Unit & Assigned value \\
\hline Pendulum mass & $\mathrm{m}$ & {$[\mathrm{kg}]$} & 1.0 \\
\hline Rotor radius & $\mathrm{R}$ & {$[\mathrm{m}]$} & 0.17 \\
\hline Pendulum length & $l$ & {$[\mathrm{~m}]$} & 0.17 \\
\hline Rotor mass moment of inertia & $\mathrm{J}$ & {$\left[\mathrm{kg} \cdot \mathrm{m}^{2}\right]$} & 0.05 \\
\hline Shaft torsional stiffness & $\mathrm{k}$ & {$[\mathrm{N} \cdot \mathrm{m} / \mathrm{rad}]$} & $23.57 \times 10^{3}$ \\
\hline Amplitude of the excitation torque & $\mathrm{T}_{\mathrm{o}}$ & {$[\mathrm{N} \cdot \mathrm{m}]$} & 50 \\
\hline Shaft viscous damping & $\mathrm{C}_{\mathrm{r}}$ & {$[$ N.m.s/rad] } & 0.6866 \\
\hline Absorber viscous damping & $\mathrm{C}_{\mathrm{a}}$ & {$[\mathrm{N} \cdot \mathrm{m} . \mathrm{s} / \mathrm{rad}]$} & 0.1 \\
\hline Gravitational acceleration & $\mathrm{g}$ & {$\left[\mathrm{m} / \mathrm{s}^{2}\right]$} & 9.81 \\
\hline Shaft Damping ratio & $\zeta$ & - & 0.01 \\
\hline
\end{tabular}

